Revue d'histoire de l'Amérique française

Q8. REVUE D'HISTOIRE DE L'AMÉRIQUE FRANÇAISE

\title{
Les neveux de Madame de Beaubassin
}

\section{Thomas Charland}

Volume 23, numéro 1, juin 1969

URI : https://id.erudit.org/iderudit/302854ar

DOI : https://doi.org/10.7202/302854ar

Aller au sommaire du numéro

Éditeur(s)

Institut d'histoire de l'Amérique française

ISSN

0035-2357 (imprimé)

1492-1383 (numérique)

Découvrir la revue

Citer cet article

Charland, T. (1969). Les neveux de Madame de Beaubassin. Revue d'histoire de l'Amérique française, 23(1), 68-91. https://doi.org/10.7202/302854ar d'utilisation que vous pouvez consulter en ligne.

https://apropos.erudit.org/fr/usagers/politique-dutilisation/ 


\section{LES NEVEUX DE MADAME DE BEAUBASSIN*}

Nombreux sont les Hertel dont les noms ont été consignés dans les annales militaires de la Nouvelle-France. L'identification des personnes n'est pas toujours facile, surtout quand la désignation en est réduite à la simple expression " le sieur Hertel". Je la tente ici pour ceux d'une branche de cette illustre famille, les Hertel de Saint-François.

Ils étaient six frères à avoir embrassé la carrière militaire, presque la seule ouverte alors aux Canadiens de naissance. ${ }^{1}$ Ils ont servi sur tous les fronts: à Louisbourg, au lac Champlain, au lac Ontario, dans la vallée de l'Ohio et finalement autour de Québec. Des six, trois sont morts au combat. Montcalm a souvent parlé de ces braves jeunes gens. Il avait un faible pour eux, et il ne s'en cachait pas: c'étaient les neveux de la dame qui l'accueillait si assidûment dans son salon, ancienne rue du Parloir, quand il se trouvait à Québec. ${ }^{2}$ Leur père, Joseph Hertel de Saint-François, était en effet le frère aîné de Pierre-Joseph Hertel de Beaubassin, époux de Catherine Jarret de Verchères, la meilleure amie de Montcalm.

Tandis que Hertel de Beaubassin avait opté pour le métier des armes, Hertel de Saint-François était demeuré sur la terre de ses parents dans l'île Saint-Jean, seigneurie de Saint-François.

* Signification des sigles employés dans cet article. AC: Archives des Colonies (Paris) - APC: Archives Publiques du Canada (Ottawa), A : Collection Bouquet (copie), B: Collection Haldimand (copie) - BRH: Bulletin des Recherches Historiques (Lévis, 1895-.........) - CMML: Collection des Manuscrits du Maréchal de Lévis, éd. H.-R. Casgrain - CMNF: Collection de Manuscrits ... relatifs à la Nouvelle-France - NYCD: Documents relative to the Colonial History of the State of New York, ed. E. B. O'Callaghan - PC: Papiers Contrecour, éd. F. Grenier - PJ : The Papers of Sir William Johnson - RAPQ: Rapport de l'Archiviste de la Province de Québec.

1 P.-G. Roy, "A propos des Hertel”, BRH, 49 (1943) : 351.

2 Montcalm écrivait à Lévis, le 11 janvier 1758: "Bourlamaque passe sa vie dans la rue du Parloir, au fond du cul-de-sac. J'en fais autant, mais c'est à l'entrée." CMML, 6: 113. L'ancienne rue du Parloir passait devant l'archevêché actuel, pour aboutir au parloir du Séminaire. P.-B. Casgrain, "La maison d'Arnoux où Montcalm est mort", BRH, 9 (1903): 37; H. Têtu, Histoire du palais épiscopal de Québec, 121. 
Dans les documents qui le concernent, il est toujours qualifié d'écuyer, sans plus, ce terme indiquant qu'il appartenait à une famille anoblie. Aussi je doute fort qu'il ait jamais été officier dans les troupes de la colonie, comme l'affirme J.-B. Melchior Hertel de Rouville dans sa généalogie de la famille Hertel. ${ }^{3}$ Il a bien été proposé pour le grade d'enseigne, vers 1737-1738. "Il sait la langue des Sauvages et est fort utile", est-il dit de lui dans un document publié par l'abbé F. Daniel ${ }^{4}$; mais la proposition n'a pas nécessairement été suivie d'une acceptation. La seule mission qui lui a sûrement été confiée est celle-ci : le 27 décembre 1735, le gouverneur Beauharnois lui ordonna de veiller à la recherche des soldats déserteurs, en se faisant aider au besoin par les capitaines, officiers et habitants des côtes de Saint-François, Yamaska et Sorel. ${ }^{5}$

Il avait épousé, à Montréal, le 14 novembre 1731, Suzanne, fille d'un riche marchand de cette ville, Maurice Blondeau, et de Suzanne Charbonnier. Eut-il des difficulté de ménage? Le 12 août 1733 , le tribunal des Trois-Rivières le condamna à une amende de 250 francs payables à Antoine Joyel dit Perrot, pour avoir battu la femme de ce dernier, Marguerite Patry. ${ }^{6}$ En 1744 , Suzanne Blondeau demanda une séparation de biens d'avec son mari. Il ressort de cette demande que Joseph Hertel, en plus d'avoir mal géré ses affaires, aurait aliéné les biens de son épouse passés en leur communauté et contracté plusieurs dettes à son insu. ${ }^{7}$ Tout cela ne les empêcha pas d'avoir de nombreux enfants: 8 garçons et 3 filles, dont quelques-uns moururent en

\footnotetext{
3 Nova Francia, 5 (1930): 160.

4 Histoire des principales familles du Canada (Montréal, 1867), 294.

5 Document aux archives de la Société historique de Chicago, publié dans le rapport des APC pour 1905, vol. 1: LXXII.

6 B. Sulte, Histoire de Saint-François-dw-Lac (Montréal, 1886), 97.

7 P.-G. Roy, Inventaire d'une collection..., 126, n. 1385. - Je vois par un contrat passé devant Joseph-Charles Raimbault, le 14 juin 1732, que Joseph Hertel avait fourni pour 231 livres et 15 sols de marchandises à Mathurin Desrosiers. Un autre contrat passé devant le même notaire, le 24 avril 1738, m'apprend qu'il avait servi d'intermédiaire au fameux John Henry Lydius pour racheter deux prisonniers des Abénakis, James Nap et Thomas Baker, au prix de 350 et de 440 livres. Par contre, c'est Suzanne Blondeau qui, à l'été de 1752 , reçoit 300 livres pour un autre prisonnier, Over Hancock, acheté des Abénakis pour la même somme (A. Baker, True Stories of New England Captives (Cambridge, Mass., 1897), $340)$.
} 
bas âge, comme on peut le voir dans le Dictionnaire généalogique de Tanguay.

Joseph Hertel mourut à Saint-François-du-Lac, le 15 décembre 1755 , peut-être victime de l'épidémie de petite vérole qui sévit cette année-là. Tanguay a pris son acte de sépulture pour celui de Joseph Hertel époux de Marie-Antoinette Bouton. Cet autre Joseph Hertel n'est mort que le 20 novembre 1768. Par ailleurs, les livres de comptes de la paroisse révèlent que Suzanne Blondeau cessa avec l'année 1755 d'entretenir, comme elle le faisait depuis 1739, la chapelle de Saint Joseph dans l'église de Saint-François. Elle alla demeurer d'abord à Montréal, puis chez son gendre Ignace de Salaberry, co-seigneur de Beauport, où elle mourut le 21 janvier 1790 . Elle avait hérité de son mari la moitié du fief de Pierreville.

Venons-en maintenant aux six enfants de ce couple qui s'illustrèrent dans les troupes de la colonie.

\section{$1^{\circ}$ JOSEPH HERTEL}

Premier né de la famille, il reçut à son baptême, le 22 octobre 1732, comme prénom celui de son père. Il n'avait pas encore seize ans, et il en était à sa sixième campagne contre les Anglais, lorsqu'il trouva la mort. Le 30 mai 1748, soixante Iroquois du Sault-Saint-Louis et du lac des Deux-Montagnes et dix Canadiens partaient de Montréal pour aller ravager les établissements de la Nouvelle-Angleterre. Ils étaient sous le commandement de Joseph Coulon de Villiers, sieur de Jumonville, qu'assistaient le jeune Hertel et un cadet du nom de l'Épervanche. Ils s'attaquèrent à trois forts, mais rencontrèrent une vigoureuse résistance de la part d'un parti ennemi. Le 26 juin, ils étaient de retour à Montréal, avec cinq chevelures, n'ayant pas eu le temps d'enlever celles de neuf à dix autres Anglais qu'ils avaient tués également. Deux Iroquois et Hertel étaient restés sur le champ de bataille. ${ }^{8}$

Les circonstances de la mort du jeune homme nous sont connues par une lettre de son père au gouverneur, le marquis de La Galissonière. Le récit s'apparente fort à ceux qu'on lit

8 CMNF, 3 : 408, 411. 
dans l'Iliade d'Homère, ce qui me fait soupçonner le missionnaire des Abénakis, le père Joseph Aubery, d'y avoir mis la main. ${ }^{9}$ Voici cette touchante lettre, telle qu'on la trouve dans les Archives des Colonies, C11A, vol. 91, fol. $145 .^{10}$
Monsieur
Permettez-moi, s'il vous plaît, de vous rendre compte de ce que j'ai appris de plus juste de la mort de mon pauvre enfant. On m'avait dit à Montréal que les Abénakis étaient les plus instruits de son sort. ${ }^{11}$ Ils m'ont assuré devant le Révérend Père Aubry que, ne voulant point abandonner son camarade iroquois tué à ses côtés, qu'il ne fût tiré du champ de bataille - quoiqu'on lui dit qu'il y avait du risque - il reçut un coup dans le haut de la cuisse gauche dont il plia seulement. Il se redressa et tira son coup à son ennemi, se mit en devoir, quoique son sang coulât beaucoup, de recharger et pendant ce temps, reçut un second coup au corps, au défaut du paleron, dont il tomba. Peu de temps après, ils l'entendirent faire un cri, comme d'un homme dangereuse- ment blessé, et enfin il en poussa un second entrecoupé qu'il ne put achever. Or, comme il était environné de Sauvages anglais, ils jugent qu'ils lui coupèrent la tête dans ce moment, parce qu'ils firent sur lui le cri de mort.
Vous voyez, Monsieur, que je n'ai plus d'espérance que dans les cinq qui me restent, que j'ai l'honneur de vous re- commander, à mesure qu'ils deviendront en état de servir. J'ai même très lieu d'être content du soin que vous en avez pris jusqu’à présent. Ils promettent beaucoup. Vous avez vu de quelle façon le cadet a pris la chose. J'ose me flatter que vous voudrez bien informer la Cour du peu de mérite d'un aîné de famille, tué à l'âge de quinze ans et demi, dans sa sixième campagne aux Anglais depuis le quinze de mars au vingt-deux de novembre. ${ }^{12}$ Sa perte, n'étant triste qu'au père et à la mère, pourrait, comme à bien d'autres, être de quelque utilité à ceux qui le suivent, s'ils marchent sur ses traces. C'est la grâce qu'attend de Monsieur le Général, dans lequel le pays trouve tant justice, celui qui a l'honneur d'être avec un très profond respect, Monsieur, votre très

${ }^{9}$ Un autre humaniste, Bougainville, a écrit dans son Mémoire sur l'état de la Nouvelle-France (1757): "On trouve dans les mœurs des Sauvages des traces des anciens usages des Grecs, principalement je crois voir dans leurs mours et coutumes guerrières celles des héros de l'Iliade et de l'Odyssée." RAPQ (1923-1924): 66.

10 L'extrait qu'en a publié Joseph Marmette dans le rapport des APC pour 1887: CI-CII, comporte quelques petites variantes.

11 Quelques Abénakis s'étaient peut-être joints à l'expédition.

12 On ne peut admettre que le jeune Hertel périt en novembre 1747, comme ce passage donnerait à croire. Il n'eut ses quinze ans et demi qu'au printemps de 1748. Par ailleurs, La Galissonière place sa mort au printemps de 1748 , tout comme le journal publié dans la CMNF. 
humble et très obéissant serviteur.

A Saint-François, ce 10ème juillet 1748.

Hertel

Le 8 novembre suivant, La Galissonière transmit cette lettre au ministre de la Marine, dont relevaient les affaires du Canada, en y ajoutant:

Je ne puis refuser au Sr Hertel de St-François de vous écrire en sa faveur et en celle de sa famille à l'occasion de la mort de son fils, que j'avais fait cadet dans les troupes au commencement de l'hiver et qui a été tué ce printemps à la sixième incursion qu'il faisait sur les terres ennemies, dans toutes lesquelles il avait donné des preuves de valeur. J'ai donné sa place à son frère, mais c'est une faible récompense, et j'espère que vous voudrez $y$ ajouter quelque grâce. Je joins ici la lettre que le père m'a écrite.

J'ignore s'il fut donné suite à cette démarche du gouverneur.

\section{$2^{\circ}$ ÉTIENNE HERTEL}

Devenu l'aîné par suite de la mort de son frère Joseph, il est souvent désigné ainsi: Hertel l'aîné. On le trouve d'abord servant en qualité de cadet dans les troupes en garnison au fort Saint-Frédéric, sur le lac Champlain. Il y fut parrain d'un enfant d'Abénakis baptisé le 14 décembre $1748 .^{13}$ On sait que, depuis 1733 environ, les Abénakis de Saint-François et de Bécancour s'étaient installés à l'embouchure de la rivière Missisquoi et qu'ils s'adressaient pour fins religieuses à l'aumônier du fort Saint-Frédéric.

Le 23 octobre de l'année suivante, le gouverneur La Jonquière lui envoya l'ordre de s'embarquer pour Louisbourg, à l'île Royale, et de servir dans l'armée à cet endroit. ${ }^{14}$ Il y fut promu enseigne en second, le ler mai 1751, puis enseigne en pied, le ler avril 1755. Un ordre du ler mai 1757 le fit revenir au Canada. ${ }^{15}$ Le ler janvier 1759 , le roi lui accordait la lieutenance, à la place du sieur Vautachen, comme l'avait proposé le gouverneur Vaudreuil. ${ }^{16}$ Entre-temps, il était allé servir au fort Michillimakinac. Il y avait laissé des fourrures, que le commandant, Louis

13 P.-G. Roy, Hommes et choses du fort Saint-Frédéric, 146, 278.

14 Le document se trouve aux archives de la Société historique de Chicago.

$15 \mathrm{AC}, \mathrm{D}^{2}$, vol. 3.

16 AC, C11A, vol. 104, fol. 439; RAPQ (1923-1924): 38. 
Liénard de Beaujeu, s'était appropriées après son départ. ${ }^{17}$ Connaissant son ascendant sur les Sauvages, on l'avait rappelé pour le mettre à la tête de partis occupés à empêcher l'armée de Wolfe de traverser la rivière Montmorency.

Le 26 juillet (1759) au matin, il traversa la rivière au gué d'en haut avec deux cents Sauvages et une trentaine de Canadiens. Les Sauvages s'embusquèrent dans les bois. Ils furent longtemps sans apercevoir l'ennemi. Las d'attendre, ils proposèrent à Hertel de leur laisser la liberté d'ouvrir le feu sur les sentinelles anglaises. Ce fut le début d'une fusillade qui dura de $11 \mathrm{~h}$. du matin jusqu'à $5 \mathrm{~h}$. du soir. Le détachement anglais qu'ils affrontèrent en était un qui escortait Wolfe dans une sortie de reconnaissance. ${ }^{18} \mathrm{Ils}$ le forcèrent de se retirer. Wolfe retourna à son camp de l'Ange-Gardien et fit envoyer des renforts. Les Sauvages en firent demander de leur côté, mais le commandant du camp français leur en refusa, disant qu'il n'avait pas ordre de se dégarnir; et le chevalier de Lévis, à qui son éloignement ne permit d'apprendre qu'en gros ce qui se passait, leur envoya l'ordre de repasser la rivière. Mécontents, ils éclatèrent en reproches à l'adresse des Français, les accusant de vouloir leur faire porter seul le poids de la guerre. ${ }^{19}$ Il y eut des pertes de part et d'autre. Wolfe avoue avoir subi les plus considérables. ${ }^{20}$

Le 15 août suivant, Montcalm ordonna à un détachement de Sauvages et de Canadiens de passer la rivière, au poste de monsieur d'Herbin, une heure avant le jour, afin de surprendre l'ennemi. Hertel devait commander les Sauvages, mais en dépendance de Pierre Le Gardeur de Repentigny, ce qui n'eut pas l'heur de lui plaire. Il venait peut-être de recevoir la nouvelle de sa promotion à la lieutenance, comme semble l'indiquer le passage suivant du Journal de Montcalm : "Cependant M. d'Artel,

17 Une sentence de la Chambre des milices de Montréal du 16 février 1761, confirmée par le général Gage le 7 mars suivant, obligea MarieGeneviève de Longueuil, épouse et procuratrice de monsieur de Beaujeu, à payer 5615 livres à la mère d'Etienne Hertel, Suzanne Blondeau. APQ, Chambre des milices de Montréal 1760-1764.

18 Knox's Historical Journal, ed. A. G. Doughty, 1: 412; "Journal du siège de Québec, annoté par A. Fauteux", RAPQ (1920-1921): 179, 233, note 257 .

19 Journal de La Pause, RAPQ (1933-1934) : 105-106.

20 Wolfe à Moncton, 27 juillet 1759, APC, Collection Northcliffe, 158. 
lieutenant de quatre jours, a trouvé dur de marcher sous ses ordres, ordres il n'y en eut jamais ici. On n'y connaît ni subordination ni discipline. Je crains fort que ce jeune homme ne dégoûte les Sauvages avant leur départ." 21

L'ordre de Montcalm fut exécuté le 17. Les Sauvages eurent le malheur de se découvrir en attaquant une maison où ils avaient vu du monde. La maison était défendue par un certain Charles Cameron, un sergent, un caporal et seize volontaires dans le régiment d'infanterie légère du général Lascelles. Sachant qu'il pourrait être promptement secouru, Cameron laissa voir deux ou trois de ses hommes pour amener les Sauvages à attaquer la maison. La tactique réussit. Le major Hussey était à peine à 150 verges de là avec son détachement. À sa vue, les Sauvages s'enfuirent à travers les bois avec tant de précipitation qu'il ne put faire tirer sur eux que six ou sept coups de fusil. ${ }^{22}$

Le Journal de Montcalm porte la trace de son mécontentement. "Qu'il est malheureux, écrit-il, de dépendre, pour des expéditions qui pourraient être importantes, d'une race indépendante et capricieuse! À quoi donc sont bons les Sauvages? À ne pas les avoir contre soi." 23 De son côté, l'auteur anonyme d'un Journal du siège de Québec s'en prend violemment aux interprètes et officiers attachés aux Sauvages:

Ces malheureux contretemps provenaient de la méchanceté des interprètes et des officiers attachés aux Sauvages qui ne pouvaient digérer le zèle et la régularité de $\mathrm{Mr}$ de Repentigny à leur faire faire le service, lequel commandait les postes avancés du côté des Bois, en outre les officiers grossiers par leur nature et dont le caractère ressemble aux Sauvages dont ils suivent les mœurs et les manières voulaient être non seulement indépendants mais même considérables en inspirant leurs sentiments aux. Sauvages, à qui ils faisaient dire ce qu'ils voulaient n'y ayant qu'eux qui entendissent et expliquassent leur langue. ${ }^{24}$

Après la capitulation de Québec (18 septembre), Hertel se retira avec les débris de l'armée de Montcalm au camp établi à l'embouchure de la rivière Jacques-Cartier.

${ }^{21}$ CMML, 7: 590.

22 Knox's Historical Journal, 2: 34; Wolfe à Moncton, 16 août 1759, APC, Collection Northcliffe, 162.

23 CMML, 7: 591.

24 APC, Collection Northcliffe, 268. 
En vue du siège de Québec projeté pour le printemps suivant (1760), Vaudreuil voulut tirer des vivres des paroisses de la rive sud, de la Pointe-Lévy jusqu'à Kamouraska. À la fin de janvier, il envoya le capitaine Jean-Jacques Gorge de SaintMartin avec un détachement de sept cents hommes prendre poste à la Pointe-Lévy, pour assurer le passage des convois qui devaient suivre la côte par terre jusque vis-à-vis la Pointe-aux-Trembles. Mais, un pont de glace s'étant formé et les Anglais ayant traversé le fleuve en force, Saint-Martin dut se retirer, après avoir perdu trente hommes. Bourlamaque jugea alors qu'il était impossible d'exécuter le projet de faire passer des vivres, et il se borna à envoyer dans les paroisses que les Anglais n'avaient point épuisées cent cinquante hommes aux ordres de l'officier Hertel, afin d'empêcher l'ennemi de pousser plus loin ses levées et de conserver les vivres jusqu'au temps où les troupes seraient devant Québec.25

Hertel établit son poste à Saint-Michel de Bellechasse, au début de mars. Le général Murray eut connaissance de la chose, et, croyant le détachement français de quatre à cinq cents hommes, il établit une redoute et une batterie de vingt-deux pièces de canon à la Pointe-Lévy. Hertel, de son côté, ne tarda pas à demander du renfort. Il avait peine à se plier à l'article de ses instructions qui lui prescrivait de s'échapper par les bois s'il était attaqué par un corps supérieur au sien. Le commandant du camp de Jacques-Cartier, le major général Jean-Daniel Dumas, aurait bien voulu lui envoyer cent hommes détachés de la Pointe-aux-Trembles. Il ne put lui en envoyer que dix. On manquait de chaussures au camp. Pour comble de malheur, les Abénakis de Saint-François et de Bécancour qui accompagnaient Hertel le quittèrent pour retourner dans leurs villages. Toutefois, il ne fut pas attaqué. Il y eut bien deux ou trois berges anglaises qui parurent vis-à-vis son poste de Saint-Michel; mais leurs occupants ne risquèrent pas le débarquement. ${ }^{26}$

25 Relation de l'expédition de Québec aux ordres de M. le chevalier de Lévis en 1760, CMML, 11: 223; CMNF, 4: 285.

${ }^{26}$ Lettres de Vaudreuil à Dumas et de Dumas à Vaudreuil, publiées dans le rapport des APC pour 1905, vol. 1, 4e partie: 5-27; Vaudreuil au ministre, 18 avril $1760, A C, F 3$, vol. 16 , fol. $24-25$. 
Le 27 avril, Hertel attaqua avec son détachement les deux redoutes anglaises de la Pointe-Lévy. Se voyant incapable de les conserver, les quatre-vingts Anglais qui les occupaient y mirent le feu et se retirèrent dans Québec. ${ }^{27}$ Hertel prit-il part à la bataille de Sainte-Foye qui eut lieu le lendemain ? Il semble que oui. On lit, en effet, dans le Registre mortuaire de l'Hôpital Général de Québec: "L'an mil sept cent soixante, le dix mai, a été inhumé dans le cimetière de cet hôpital le corps d'un soldat du parti de Monsieur Hartel, tué par un (sic) de nos sentinelles au siège de Québec; il s'est confessé et a reçu le sacrement d'extrême-onction; en foi de quoi j'ai signé

Rigauville, ptre chanoine. ${ }^{28}$

Au début de juin, Vaudreuil ordonna au major Dumas de faire passer deux petits détachements sur la rive sud du fleuve, l'un en bas de Sainte-Croix et l'autre en haut de Lotbinière, avec mission de rassurer les habitants, de leur faire prendre les armes pour en imposer à l'ennemi, et de s'opposer à ses incursions. Dumas se trouva embarrassé quant au choix des officiers à mettre à la tête de ces détachements. Les deux seuls qu'il croyait aptes à cette mission étaient Audet de Bailleul et Hertel; mais pour les détacher de la garnison de Repentigny il lui aurait fallu se brouiller avec ce dernier, qui n'aimait pas qu'on diminue son monde. Il demanda donc à Vaudreuil de lui envoyer un ordre pour Hertel. Il reçut de lui cette réponse: "C'est à vous, Monsieur, à destiner les officiers que vous croirez les plus propres pour commander à la côte sud. J'écris en conséquence à $M$. de Repentigny que vous serez obligé d'avoir recours à M. Hertel et de prendre même un autre officier de sa garnison. M. de Bayeul étant malade, il ne saurait avoir cette destination." Dumas choisit Saint-Blin et Hertel, le premier pour le bas de Sainte-Croix et l'autre pour le haut de Lotbinière. Ils se rendirent à leurs postes le 24 juin. ${ }^{29}$

Le général Murray s'embarqua à Québec, le 14 juillet, avec 2500 hommes de troupes, et commença à remonter le fleuve à

27 Journal du curé Récher, BRH, 9 (1903) : 143.

28 RAPQ (1920-1921): 276.

29 APC, rapport pour 1905, vol. 1, 4e partie: 33-36, 47. 
destination de Montréal. Sur son parcours, il faisait descendre des officiers et des soldats pour désarmer les habitants des paroisses et leur faire prêter le serment d'allégeance au roi d'Angleterre. Ce qui se passa à Lotbinière nous est connu par le Journal de Murray lui-même et celui du capitaine Knox, ${ }^{30}$ et par une lettre de Vaudreuil au ministre de la Marine, du 6 août $1760 .{ }^{31}$ Le 18 juillet, un détachement de cent hommes, que Murray avait fait débarquer la veille au soir, se mit en marche à $3 \mathrm{~h}$. du matin à travers les bois, tandis qu'un autre détachement de deux cents hommes occupait la route à deux endroits. Hertel n'avait que quarante hommes sous ses ordres. Craignant d'être tourné, il chercha à tourner lui-même les Anglais. Les deux partis se rencontrèrent. Après une première décharge, la petite troupe de Hertel prit la fuite à travers les bois. Hertel fut blessé et fait prisonnier avec trois ou quatre de ses hommes. On les amena à bord de la frégate Diana. Hertel succomba à ses blessures, le soir même. Le lendemain, son corps fut porté à Deschambault, de l'autre côté du fleuve, à bord d'une berge arborant pavillon blane, pour être remis au commandant de Jacques-Cartier, Monsieur de Repentigny, avec une lettre de Murray à ce dernier. Dans cette lettre, le général anglais affirmait savoir de très bonne part que Hertel s'était presque toujours trouvé à la tête des Sauvages et qu'il se plaisait plus à faire le guerre en parti bleu qu'en officier. En dépit de cela, il renvoyait son corps pour qu'on lui rende les derniers devoirs.

Hertel fut inhumé le même jour, dans le cimetière du CapSanté, sous le nom de Jean, comme le révèle le registre de cette paroisse.

L'an mil sept cent soixante le dix-neuf de juillet par nous prêtre curé de cette paroisse de la Ste Famille du Cap-Santé, a été inhumé monsieur Jean Artel lieutenant des troupes de la marine, mort d'hier de la main de l'ennemi et n'ayant

${ }^{30}$ L'un et l'autre édités avec des notes par A. G. Doughty dans la collection de la Champlain Society sous le titre Knox's Historical Journal, (Toronto, 1914). - Dans le tome 2, p. 472, note 2, Doughty dit que notre Hertel était au fort Frontenac en 1751. C'est une erreur. C'est Hertel de Beaubassin qui y était, d'après l'Extrait général des revues de compagnies entretenues en la Nouvelle-France $1751, \mathrm{AC}, \mathrm{D}^{2}$, vol. 48.

31 A.C, F3, vol. 16, fol. 101-103. 
par conséquent pu recevoir les derniers sacrements. Ont assisté à la sépulture plusieurs de cette paroisse qui ont déclaré ne scavoir signer, de ce enquis suivant l'ord.

J. Fillion, ptre

Étienne Hertel n'était âgé que de vingt-cinq ans, ayant été baptisé le 9 novembre 1734 .

\section{$3^{\circ}$ PIERRE-ANTOINE HERTEL}

Il commença son service dans les troupes de la marine à Louisbourg, où il était allé rejoindre son frère aîné Étienne. Pour le distinguer de ce dernier, on l'appela Hertel le cadet. Et on continua de l'appeler ainsi, même après qu'il eut été promu enseigne en second, le ler avril 1754.32. Quant à lui, après son retour, il se fit appeler Hertel Sorel de Louisbourg. Passé au Canada par congé, il y fut retenu par ordre du gouverneur et envoyé au camp de Carillon, sur le lac Champlain.

Le 23 juillet 1756, il partait à l'avant-garde d'un détachement commandé par le capitaine Antoine de LaCorne la Colombière et composé de soixante et onze Français et Canadiens et de cinquante-six Sauvages, pour aller frapper un coup au fort George, au fond du lac Saint-Sacrement. Il s'agissait de faire des prisonniers, dont on tirerait des nouvelles, et d'inquiéter l'ennemi pour l'empêcher de porter son attention vers Chouaguen, dont Montcalm s'en allait faire le siège. Le détachement était de retour le 26. Il avait levé quatre chevelures et tué une trentaine d'Anglais aux environs du fort. Il avait dû se retirer, en voyant sortir du camp un gros détachement, avec lequel, du reste, il fusilla environ une demi-heure dans les bois. ${ }^{33}$ Lévis écrivait à Vaudreuil, le 2 août suivant: "Tout ce détachement se loue beaucoup des officiers qu'il avait avec

${ }^{32} \mathrm{AC}, \mathrm{D}^{2}$, vol. 3. - Dans son ouvrage sur les Chevaliers de SaintLouis en Canada, A. Fauteux a écrit que Pierre-Antoine Hertel fut fait enseigne en second en 1751 et enseigne en pied en 1755. Ces dates s'appliquent plutôt à son frère Etienne. Toujours d'après Fauteux, qui s'appuie sur Alex. Mazas (Histoire de l'Ordre royal et militaire de Saint-Louis, $2 \mathrm{e}$ éd. Paris, 1860-1861, 2: 231), Pierre-Antoine était cadet dès 1747. Or, on se rappelle qu'après la mort de Joseph, en 1748, La Galissonière avait donné sa place de cadet à son frère Etienne.

33 Lettres de Lévis, CMML, 2: 26, 29, 31-33, 39-40, 55; Journal de Lévis, CMML, 1: 47-48, 53. 
lui, particulièrement de M. Artel Saurel, officier de Louisbourg, qui faisait son avant-garde." ${ }^{34}$

Le 28 août, nouvelle tentative pour savoir ce qui se passait au camp de l'ennemi. Lévis envoya dans ce but un parti de dix-sept Sauvages, Hurons et Abénakis, sous la conduite de Hertel le cadet, l'officier de leur choix. ${ }^{35} \mathrm{Ils}$ n'osèrent aller plus loin que le Pain-de-Sucre, à une lieue et demie environ des îles du lac Saint-Sacrement. Il leur parut que ces îles étaient occupées en force par les Anglais. ${ }^{36}$

$\mathrm{Au}$ printemps de 1757 eut lieu une première tentative pour s'emparer du fort George. Un détachement de 1600 hommes, commandé par Rigaud de Vaudreuil, gouverneur des TroisRivières, partit de Carillon le 15 mars et alla camper à la droite $\mathrm{du}$ lac Saint-Sacrement. Le lendemain, au point du jour, on envoya en découverte soixante-quinze Sauvages et vingt-cinq Français que commandaient Hertel de Louisbourg et AntoineCharles de Saint-Simon. ${ }^{37}$

Quelques semaines plus tard, le commandant du camp de Carillon, Bourlamaque, confiait à Hertel et à un nommé Laframboise une autre expédition de reconnaissance, qui les conduisit jusqu'aux environs du fort Lydius. Ils y virent des troupes anglaises en route pour le fort George. De Montréal, Vaudreuil écrivait à Bourlamaque, le 4 juin: "J'ai beaucoup de confiance à la nouvelle découverte dont vous avez chargé les Sieurs Hertel et Laframboise. Je suis bien aise que vous soyez satisfait du zèle de ce dernier. Il ne faut rien moins, Monsieur, pour me disposer à lui procurer son avancement, quoiqu'il ne soit pas un des anciens cadets." ${ }_{38}$ Rien pour Hertel. Celui-ci fit-il part d'une certaine indifférence de Vaudreuil à son endroit à Montcalm, qu'il rencontra à Montréal ? On sait qu'il y avait souvent mésentente entre Vaudreuil et Montcalm. Toujours est-il que, cinq jours plus tard, Montcalm écrivit au

34 CMML, 2: 33.

35 Lettres de Lévis, CMML, 2: 69, 75.

36 "Journal d'Avène des Méloizes", RAPQ (1928-1929): 12.

37 Journal de Montcalm, CMML, 7: 176; "Journal de Bougainville", RAPQ (1923-1924) : 256

38 APC, Collection Bourlamaque, vol. 2: 74. 
même Bourlamaque: "M. Hertel de Louisbourg, qui vous remettra ma lettre, mon cher Bourlamaque, est un joli sujet, je vous le recommande. Si vous avez occasion, employez-le, et s'il fait bien, comme je n'en doute pas, écrivez à $M$. le Général... M. Hertel compte avoir beaucoup de crédit avec les Abénakis, qui sont en petit nombre à votre camp." 39

La faveur dont Montcalm entourait Hertel ne s'explique pas seulement par les qualités qu'il reconnaissait au jeune militaire. Il y avait que ce dernier était en outre le neveu de madame de Beaubassin. La séduisante Catherine avait dû parler de son neveu au général, au cours de leurs fréquentes conversations dans le salon de la rue du Parloir à Québec. Le 20 de ce même mois de juin, de Saint-Jean Montcalm écrivait encore à Bourlamaque: "[Pour vous seul]. Je vous envoie des lettres pour votre camp, et une pour M. Hertel de Louisbourg. Je vous recommande tout ce qui est Hertel: ce sont les neveux d'une dame que vous aimez, et qui vous est fort attachée; car elle me parle souvent de vous, avec les plus grands éloges, mais vous avez passé sur le grand banc." 40

Bourlamaque ne tarda pas à employer le "joli sujet" que lui avait recommandé Montcalm. Le 30 juin, il fit partir en découverte un détachement de deux cent vingt-cinq Sauvages sous la conduite de trois officiers: Hertel de Louisbourg, un de ses frères et Godefroy de Linctot, et six cadets. En entrant dans la Rivière-au-Chicot, qui est étroite et escarpée de rochers, le détachement fut salué par une mousquetade qui tua un cadet, le sieur de Richerville, et blessa à mort quelques Sauvages. Il alla se mettre à l'abri sur la rive opposée et y passa la nuit. $\mathrm{Au}$ point du jour, il traversa la rivière et délogea l'ennemi, se mit à sa poursuite et captura deux Moraïgans et un Anglais grièvement blessé. Les Sauvages déchiquetèrent un des Moraïgans, et, l'ayant trouvé bon, le mangèrent. Les dépositions des deux autres prisonniers confirmèrent les dernières nouvelles qu'on avait de la force des ennemis. ${ }^{41}$

${ }^{39}$ CMML, 5: 157-158; APC, Collection Bourlamaque, vol. 1: 81-84.

40 CMML, 5: 173.

41 Journal de Montcalm, CMML, 7: 225; "Journal de Bougainville", RAPQ (1923-1924) : 270 . 
Au siège du fort George (ou William-Henry), en août 1757, l'armée de Montcalm comprenait 1800 Sauvages, dont 245 Abénakis de Saint-François, de Bécancour, de Missisquoi et de Panaouamské en Acadie. Ces Abénakis avaient pour officiers le chevalier Joseph-Claude Boucher de Niverville et notre jeune Hertel. ${ }^{42}$ On sait quel affreux carnage se produisit le lendemain de la capitulation du fort. Les Sauvages, assoiffés de pillage, se ruèrent sur la colonne de 2000 Anglais qui venait de se mettre en marche pour le fort Edward, escortée par 200 soldats et officiers français. Enivrés avec du rhum et autres spiritueux trouvés au camp dans les bagages des Anglais ou donnés par ceux-ci dans le but de se les rendre favorables, ils massacrèrent une cinquantaine de soldats, de femmes et d'enfants et firent de cinq à six cents prisonniers. Le tumulte fit accourir Montcalm, Lévis, Bourlamaque, les officiers, les interprètes et les missionnaires. Au risque de leur vie, ils arrachèrent plus de quatre cents prisonniers des mains des Sauvages. ${ }^{43}$ Les autres prisonniers, que les Sauvages n'avaient pas voulu rendre et qu'ils avaient amenés à Montréal, furent rachetés par Vaudreuil et escortés jusqu'en Nouvelle-Angleterre.

A-t-on eu raison de soupçonner les officiers et les interprètes des Sauvages de les avoir en sous-main encouragés à piller les bagages de l'ennemi ? ${ }^{44}$ Il est certain que ce sont les Abénakis de Panaouamské qui ont déclanché l'assaut, pour venger plusieurs de leurs guerriers traîtreusement mis à mort, l'hiver précédent, dans les forts anglais de l'Acadie. ${ }^{45}$ Il est certain aussi que les officiers et les interprètes des Sauvages n'étaient pas sur la scène au moment de l'assaut: la colonne des prisonniers et son escorte étaient parties sans eux, contrairement à ce qui avait été convenu.

Rassurés du côté du lac Champlain, les autorités du pays songèrent à renforcer la région de la Belle-Rivière (Ohio), elle ville", 287 .

42 NYCD, 10: 620; Journal de Montcalm, 264; "Journal de Bougain-

43 Montcalm à Webb, CMNF, 4: 114, 117.

44 "Journal de Bougainville", 304; Correspondance de Bougainville, Quimper, 1932-1935, 2: 11.

$45 \mathrm{~T}$. Chapais, Le marquis de Montcalm, 280, note 1. 
aussi menacée. Le 21 septembre 1757, Vaudreuil pria Bourlamaque "de vouloir bien faire descendre à Montréal par la plus prochaine occasion M. Hertel Sorel officier de Louisbourg et M. Dusablé. Je souhaite qu'ils ne tardent pas à arriver parce que je les ai destinés à aller hiverner dans nos postes de la Belle Rivière et qu'ils n'ont pas de temps à perdre pour s'y rendre." ${ }^{46}$

On se rendait à la Belle-Rivière par le lac Ontario, le portage de Niagara et le lac Érié. De là on pouvait prendre le portage de la Presqu'île à la Rivière-aux-Bœufs, ou bien celui de la petite rivière Chatakoin au lac du même nom (aujourd'hui Chautauqua, N.Y.). C'est cette dernière route qu'avait suivie le baron de Longueuil pour aller en Louisiane, en 1739, et Céloron de Bienville, dans sa campagne de 1749. En 1753 , le président du conseil de Marine avait dissuadé le gouverneur Duquesne de construire trois forts: au portage de Chatakoin et aux deux villages des Chaouanons, Chiningué et Sonioto. On se contenta d'y poster des garnisons, surtout à Chatakoin, important entrepôt de vivres et de munitions.

Je vois par le Journal de Montcalm et par une lettre de ce dernier à Bourlamaque, du 12 mai 1759, que Hertel, simple enseigne, était commandant à Chatakoin et qu'il s'occupait de rallier à la cause française des nations sauvages, comme les Chaouanons (Shawanee), les Têtes Plates et les Chats. ${ }^{47}$

À l'été de 1760, les garnisons de tous les postes de la région de la Belle-Rivière se retirèrent au Détroit, en emportant artillerie, munitions, provisions et marchandises, autant qu'elles purent en emmagasiner dans les bateaux. Le 20 juin, Vaudreuil écrivait au ministre Berryer que Hertel tenait encore bon au village de Sonioto, sur la rivière Scioto, où il l'avait placé. ${ }^{48}$ Toutefois, Hertel ne tarda pas à se replier lui-même sur le Détroit. On voit, par les registres du fort Pontchartrain de cet endroit, que le 27 juillet il servit de parrain au fils de ClaudeThomas Gouin, aide-major des milices, et de Marie Cuillerier. Le 21 septembre suivant, il était encore parrain, cette fois d'une esclave d'Antoine Cuillerier, la chicachase Marie-Louise.

${ }^{46}$ APC, Collection Bourlamaque, vol. 2 : 178.

47 CMML, $7: 518$; 5: 311-312.

48 NYCD, 10: 1093; Wisconsin Historical Collections, 18 (1908) : 218. 
A la fin de novembre, le major Robert Rogers arriva de Montréal avec son détachement pour prendre possession du Détroit. Le commandant, François-Marie Picoté de Belestre, et les officiers de la garnison se constituèrent prisonniers de guerre, aux termes de la capitulation de Montréal, et furent acheminés par le fort Pitt jusqu'à New-York, où on les embarqua pour l'Angleterre. Quant à Hertel, il se constitua lui aussi prisonnier de guerre; mais le 7 décembre, Rogers l'envoya accompagner le lieutenant Alexander McGee (McKee) au village des Chaouanons sur la rivière Scioto, ${ }^{49}$ pour y prendre le reste de la troupe française qui s'y trouvait et la ramener à Pittsburg. ${ }^{50}$ Hertel et McGee n'arrivèrent à Pittsburg que le 12 février suivant (1761), et avec six hommes seulement sur dix-huit: quelquesuns avaient été laissés au village malades, d'autres avaient déserté durant le voyage. Hertel lui-même arriva malade. ${ }^{51}$ Le 6 mars, le colonel Henry Bouquet ordonna à un de ses officiers du fort Pitt de se mettre en marche avec les prisonniers: Hertel, trois soldats des troupes françaises et cinq miliciens, pour aller les remettre au général Moncton, à Philadelphie, ou à New-York, s'il se trouvait en cette dernière ville. ${ }^{52}$

Conduit en Angleterre, Hertel y demeura prisonnier pendant quelques années. Dans une lettre qu'il écrivit au président du conseil de Marine, le 6 février 1766, il plaida ces circonstances pour n'avoir pas déclaré dans les délais prescrits les états de fournitures faites par lui en Canada, pour lesquelles il voulait se faire rembourser. Le président pria $\mathrm{M}$. de Fontanieu, Conseiller d'Etat chargé de la liquidation de la dette du roi pour le Canada, de faire tout ce qui serait possible en sa faveur. ${ }^{53}$ Cette même année, Hertel fut fait lieutenant dans la légion de l'Île-de-France (ancien nom de l'île Maurice), et, en 1770, il

49 "At the Shawanoes town on the Ohio", écrit Rogers dans son Journal. G. Frégault (Le grand marquis, 341 ) place ce village au confluent du Scioto et de l'Ohio. Par ailleurs, sur la carte de Thomas Hutchins, reproduite par F. Parkman dans son ouvrage The Conspiracy of Pontiac, le Lower Shawanese Town est situé en amont du Scioto. 50 APC, A 27: 85.

51 APC, A 8: 250; "George Croghan's Journal", The Pennsylvania Magazine of History and Biography, 71 (1947): 399.

52 APC, A 23-1: 75.

53 AC, B 125, fol. 38 . 
fut promu capitaine. Ses services lui valurent le titre de Chevalier de Saint-Louis, le 1er mai $\mathbf{1 7 7 5}$. Le mémoire de proposition rappelle qu'il avait fait la guerre du Canada et avait été blessé de deux coups de feu. ${ }^{54}$

Il épousa, à l'Île-de-France, Geneviève-Catherine-Thérèse Panon. Il vivait encore en 1813 , au moment où J.-B. Melchior Hertel de Rouville rédigeait sa généalogie de la famille Hertel. ${ }^{55}$ Il avait écrit, en 1779: "Né au Canada, lorsque ce pays fut pris, je ne balançai pas à quitter ma patrie, mes parents, mes espérances, rien ne put me distraire de l'amour que je portais au Roi." 56

\section{$4^{\circ}$ JOSEPH-HIPPOLYTE HERTEL}

Il se faisait appeler le Chevalier Hertel. On sait que "sans appartenir à aucun ordre de chevalerie, les cadets de familles nobles, au Canada, se promouvaient volontiers à la dignité de Chevalier." 57

Il commença sa carrière militaire vers l'âge de quinze ans, dans la région de la Belle-Rivière. Le 26 février 1754, La Chauvignerie, commandant du poste de Chiningué (aux environs du fort Duquesne), l'envoya porter un message à celui du fort de la Rivière-aux-Bœufs, Jacques le Gardeur de Saint-Pierre. Il écrivait à ce dernier: "L'occasion d'un Sauvage sur lequel je comptais pour vous porter celle que je vous ai écrite du 23e de ce mois m'ayant manqué, je prends de parti d'envoyer le Chevalier Hertel avec le Sr Baby qui m'assure savoir les chemins d'ici chez M. de Joncaire." 58

Hertel prit part à l'éclatante victoire de la Monongahéla sur l'armée du général Braddock, le 9 juillet 1755. Il était cadet de la brigade de Jean-Jacques Gorge de Saint-Martin, dans le détachement de Daniel-Hyacinthe Liénard de Beaujeu. ${ }^{59}$ Quelque temps après la bataille, le commandant en chef de la Belle-Rivière,

54 Alex Mazas, op. cit., 231.

55 Nova Francia, 5 (1930): 160.

56 Robert de Roquebrune, Les Canadiens d'autrefois, 2: 82.

57 Louvigny de Montigny, "Le Lorimier et le Montigny des Cèdres", BRH, 47 (1941) : 35.

68 PC, 104. - Joncaire se trouvait au poste de Venango, à l'embouchure de la Rivière-aux-Boufs. $\mathrm{PC}, 93$ note 1,188 note 1 .

69 PC, 344. 
Claude-Pierre Pécaudy de Contrecœur, l'envoya au fort de la Rivière-aux-Bœufs aider le commandant, Duverger de SaintBlin, au portage et dans les divers travaux dont cet officier était chargé. ${ }^{60}$

Notre cadet fut promu enseigne en second en $1756,{ }^{61}$ et, le 1er janvier 1759, sur la recommandation de Vaudreuil, enseigne en pied, à la place du Sieur Cournoyer, qui devenait lieutenant. ${ }^{62}$ Il semble qu'il ait été ramené du front de la BelleRivière à celui du lac Champlain. C'est peut-être de lui qu'il est question dans ce passage d'une lettre de Montcalm à Lévis, du 20 juillet 1756: "M. Hertel, ci-devant cadet, aura le traitement comme officier, du jour qu'il est." "63 Par ailleurs, dans sa lettre du 20 juin 1757 à Bourlamaque, Montcalm parlait des neveux de madame de Beaubassin; et, le 30 du même mois, Bourlamaque envoyait en découverte un détachement de Sauvages sous la conduite de trois officiers, dont Hertel de Louisbourg et "un de ses frères".

En 1759, alors qu'Étienne était dans la région de Québec et Pierre-Antoine dans celle de la Belle-Rivière, Hippolyte servait à Carillon, où on l'employait à faire des découvertes avec des partis de Sauvages. Celle qu'il entreprit au début de mars, aux environs du fort Lydius, causa de vives inquiétudes au commandant de Carillon, monsieur d'Hébécourt. Ce dernier craignait que l'ennemi ne lui eût coupé le chemin ${ }^{64}$. Le ler juin, Hertel repartait avec Langy de Montégron et trente ou quarante Sauvages pour aller faire des prisonniers. Ils étaient de retour le 9. Langy revint fort mécontent de n'avoir pas atteint son objectif. Les Sauvages, au lieu de le conduire entre le fort Lydius et Sarasto, l'avaient mené aux environs de Corlar, où ils ne capturèrent qu'un jeune paysan assez mal instruit et une petite fille, dont la mère avait été tuée alors qu'elle se sauvait à cheval. ${ }^{65}$

60 Contrecœur à Vaudreuil, 3 août 1755, PC, 407.

61 APC, rapport pour 1886: CLXXVIII.

62 AC, D2, vol. 3 ; RAPQ (1923-1924): 38; APC, Collection Bourlamaque, vol. $6: 340$.

63 CMML, $6: 27$.

64 Hébécourt à Montcalm, 5 mars 1759, APC, Collection Bourlamaque, vol. 4: 319; Journal de Lévis, CMML, 7: 170.

65 "Journal d'Avène des Méloizes", RAPQ (1928-1929) : 32-34. 
Après la conquête du Canada par l'Angleterre, le Chevalier Hertel passa en France. Il figure, comme enseigne, sur la liste des officiers arrivés au Havre, le ler janvier 1762, par le Moulineux venant de Québec. ${ }^{66}$ Il ne séjourna pas longtemps en France, car, en 1763, il obtenait son passeport pour revenir au Canada ${ }^{67}$ Il s'établit à Montréal.

Il ne se fit pas faute de frayer, sinon de pactiser, avec les nouveaux maîtres du pays. Son nom apparaît sur une liste de chefs de famille protestants de Montréal signée par Murray, le 26 octobre 1764. ${ }^{68}$ Comme plusieurs autres Canadiens, il s'affilia à la Grande Loge maçonnique du district de Montréal, fondée en $1762^{69}$ : ce qui dut lui faciliter l'obtention du grade de capitaine et d'un emploi gouvernemental, celui d'officier des Indiens. Il n'est pas surprenant qu'il soit allé contracter mariage devant un ministre protestant, David-Chabrand Delisle, à la Christ Church de Montréal. Le 3 août 1767, il y épousait MarieAnne Lecomte-Dupré, âgée de 26 ans, fille de feu Jean-Baptiste Lecomte-Dupré et de Marie-Anne Hervieux. E.-Z. Massicotte suppose qu'après une forte opposition de sa mère, la demoiselle se serait évadée de la maison et serait allée se marier à l'église anglicane. Toujours est-il que, trois semaines plus tard, le 24 août, le curé de l'église Notre-Dame, l'abbé Jolivet, donnait au couple la bénédiction nuptiale, cette fois "du consentement de la mère de l'épouse". ${ }^{70}$

Auparavant, en 1764, Hertel avait servi au cours de la campagne militaire menée contre le chef outaouais Pontiac et les tribus indiennes des pays d'En-Haut qu'il avait soulevées contre les Anglais. ${ }^{71}$ Pour aider à réprimer cette révolte, le gouverneur Murray leva un corps de trois cents volontaires

${ }^{66} \mathrm{~A} C, \mathrm{D}^{2}$, vol. 48 .

67 F. Daniel, Histoire des principales familles du Canada, 2: 369, 371. 68 APC, Q. 2: 335; BRH, 39 (1933) : 158.

69 APC, rapport pour 1944: XXXII; BRH, 52 (1945) : 179, 233-235.

70 BRH, 32 (1926) : 536-537.

71 C'est sans doute parce que Pierre-Antoine Hertel avait séjourné dans la même région, quelques années auparavant, que $R$. G. Thwaites l'a confondu avec son frère Hippolyte, dans une note des Wisconsin Historical Collections, 18 (1908) : 218. Il a fait des deux frères un seul et même personnage, Pierre-Antoine, chevalier de Hertel. Il a été suivi en cela par l'éditeur du "George Croghan's Journal 1759-1763", dans The Pennsylvania Magazine of History and Biography, 71 (1947): 399. 
canadiens, et, de son côté le surintendant William Johnson en leva un d'Indiens de l'est. On comptait sur le ralliement des Canadiens et des Indiens pour faire impression sur les tribus révoltées, en leur montrant qu'elles n'avaient aucun secours à attendre d'eux. Un contingent de soixante Iroquois de Caughnawaga partit le 17 mai 1764 et arriva au Fort Johnson le 29. Un second partit le 5 juin pour Oswego. ${ }^{72}$ Les deux rejoignirent à Niagara l'armée du général John Bradstreet, partie d'Albany. Hertel fut placé à leur tête.

En se rendant au Détroit, Bradstreet fit la paix avec les tribus des Delawares (Loups) et des Shawanees (Chaouanons). Mais le commandant en chef, le général Gage, désavoua l'arrangement, jugeant les conditions imposées par Bradstreet trop bénignes. Bradstreet se trouvait à Sandusky lorsqu'il reçut la condamnation de Gage. Il écrivit alors au colonel Henry Bouquet, se disant incapable de demeurer plus longtemps en territoire indien et annonçant son intention de se retirer avec son armée. C'est Hertel qui fut chargé d'aller, avec son parti d'Iroquois, porter la lettre au colonel Bouquet, à son camp sur la rivière Muskingum. ${ }^{73}$

Le colonel Bouquet prit alors l'affaire en mains. Les Senecas, les Delawares et les Shawanees se soumirent; mais il leur signifia qu'ils devaient envoyer à Johnson des députés pour conférer avec lui au sujet des conditions de paix, et des otages pour garantir leur maintien dans la bonne foi. C'est encore Hertel qui fut chargé d'escorter les députés et les otages avec son parti d'Iroquois. Bouquet recommanda au capitaine David Hay, du Fort Pitt, de veiller aux besoins de M. Artel, "gentilhomme canadien, dénué de tout". ${ }^{74}$ On espérait que Hertel, parti le 1er décembre, serait à Johnson Hall vers la Noël. Il n'y arriva que le 19 février suivant (1765), après une marche épuisante sur une épaisse couche de neige. ${ }^{75} \mathrm{Au}$ cours d'une conférence

72 Burton à Haldimand, 16 mai 1764, APC, B 9: 72-73; Burton à Johnson, 6 juin 1764, PJ, 11: 207, 219.

73 F. Parkman, The Conspiracy of Pontiac, $2: 237$.

74 Bouquet à Hay, 18 novembre 1764, APC, A 21: 75

75 Bouquet à Johnson, 25 janvier 1765, APC, A 23-2: 489; Johnson à Bouquet, 20 février 1765, et à Gage, 22 février 1765, PJ, 11: 585, 592. 
tenue les 25 et 26, Johnson félicita les Iroquois de leur bonne conduite durant la campagne de Bradstreet, et il leur exprima sa satisfaction du rapport de Bouquet sur le concours qu'ils lui avaient apporté sur la Muskingum et au Fort Pitt. ${ }^{76}$

Hertel était de retour à Montréal à la mi-avril. ${ }^{77}$ Dans une lettre à Bouquet, du 17 décembre précédent, Johnson avait écrit: "Mr. Artel was one of my officers for the campaign. I know he is capable of some service amongst the Indians of Canada, and I am glad he have merited your recommandation, as it will induce me to employ him when an opportunity offers."78 Le 7 juin 1769, le gouverneur Carleton nommait Hertel interprète des Abénakis de Saint-Régis. ${ }^{79}$

Après la destruction de leur village par le major Rogers, le 4 octobre 1759, quelques Abénakis de Saint-François étaient allés demander asile et protection à la mission iroquoise de Saint-Régis. Lorsque la mission de Saint-François fut rétablie par le père Charles Germain, s.j., à la fin de 1767, les Abénakis furent priés par les chefs de Saint-Régis de s'en retourner chez eux, et, comme ils s'y refusaient, les Iroquois accusèrent Hertel de les encourager à rester, dans le but de continuer de faire la traite des fourrures avec eux. Lors d'une réunion tenue au Sault-Saint-Louis, le 21 août, 1769, les Iroquois se plaignirent à l'agent Daniel Claus que les Abénakis détruisaient leurs trappes à castor et envahissaient les terrains de chasse des Six-Nations, au risque de les entraîner dans un conflit avec elles. Claus leur répondit que, dans une conversation qu'il avait eue avec Carleton, ce dernier avait manifesté sa résolution de laisser les Abénakis à Saint-Régis, et avait même dit que les Iroquois ne devaient pas se considérer maîtres et propriétaires des terres sur lesquelles ils vivaient. ${ }^{80} \mathrm{Au}$ mois d'août de l'année suivante, les Abénakis, accompagnés de Hertel, se rendirent à Johnson Hall, sur la rivière Mohawk. Ils obtinrent du surintendant un délai de deux ans pour leur permettre de vendre leurs maisons

76 PJ, 11 : 605-607.

77 PJ, 11: 699.

78 APC, A 21: 114 .

79 APC, Claus Papers, vol. 1. 136.

80 PJ, 7 : 110-111, 127-128. 
à Saint-Régis et aller s'établir à la rivière Missisquoi. ${ }^{81}$ Mais le 2 octobre, lors d'une réunion au Sault-Saint-Louis, les chefs des deux villages iroquois décidèrent que les Abénakis et Hertel devaient se retirer sans délai. Claus conseilla à Hertel de se désister. ${ }^{82}$

Lors de l'invasion du Canada par les troupes américaines, en 1775 , le Chevalier Hertel servit en qualité de volontaire sous les ordres de Picoté de Belestre et de Longueuil. Il fut fait prisonnier lors de la capitulation du fort de Saint-Jean, le 5 novembre. ${ }^{83}$ Un placet signé par quatorze prisonniers, dont Hertel, fut remis au général Richard Montgomery, lui disant qu'ils n'étaient que des volontaires et lui demandant de ne pas les exiler dans les Colonies, mais de les conduire plutôt dans les camps que les Américains avaient à Laprairie, Longueuil et Chambly, et, sitôt que Montréal serait tombé, de les libérer sur promesse de ne pas reprendre les armes, afin que leurs familles et leurs affaires n'aient pas trop à souffrir de leur éloignement. Montgomery fit remarquer qu'il y avait des officiers parmi les signataires; et, comme un des porteurs, le Sieur Gaucher, lui affirmait respectueusement que non, il répliqua: "Ne m'en imposez point, je connais M. Hertel, compris en ce placet, j'ai fait campagne avec lui, il y a neuf ou dix ans, je ne puis vous accorder votre demande." Il faisait allusion à la campagne de 1764 contre Pontiac, au cours de laquelle il avait rencontré Hertel, probablement à Sandusky. Il finit néanmoins par se laisser fléchir et envoya treize des prisonniers à Chambly. Le quatorzième, Hertel, dut prendre le chemin des Colonies. ${ }^{84}$ Libéré lors d'un échange de prisonniers, il revint à Montréal, mais ne prit aucune part au reste de la guerre contre les Américains.

Il décéda à Montréal et fut inhumé dans la chapelle de Sainte-Anne de l'église Notre-Dame, le 10 août 1781. Il fut décidé, pour raison d'économie, de ne pas lui donner de successeur dans le Département des Sauvages ${ }^{85}$. Sa veuve s'adressa

81 Claus à Johnson, 12 septembre 1770, PJ, 7: 897-898.

82 PJ, 7: 922, 959 .

83 BRH, 12 (1906) : 317; 31 (1925) : 373.

84 "Journal tenu pendant le siège du fort Saint-Jean, en 1775, par feu M. Foucher, ancien notaire de Montréal", BRH, 40 (1934) : 144, 212-215. 85 Mathews à Campbell, 20 août 1781, APC, B 113: 149. 
par deux fois, le 28 octobre 1781 et le 27 juin 1782, au général Haldimand pour réclamer une pension viagère. "M. Hertel, lui écrivait-elle, ne m'a laissé pour toute fortune qu'un fils qui n'attend que l'âge pour offrir ses services à son Roi." ${ }^{86}$ Ce fils, Hippolyte, fut plus tard lieutenant des Volontaires Royaux. Lui-même eut deux fils, Jean-Hippolyte et Daniel, qui furent officiers des Voltigeurs pendant la guerre de 1812-1815. ${ }^{87}$

\section{$5^{\circ} \cdot 6^{\circ}$ LOUIS-JOSEPH ET JEAN-BAPTISTE HERTEL}

Je suis très peu renseigné sur ces deux derniers fils de Joseph Hertel et de Suzanne Blondeau, baptisés, l'un le 12 septembre 1741, l'autre le 10 mai 1745.

Un Louis Hertel fut fait cadet dans les troupes par un brevet signé par le gouverneur Duquesne et daté de Québec, le 23 octobre $1753 .{ }^{88} \mathrm{Il}$ fut promu enseigne en second, en $1757 . .^{89}$ Il s'agit plus probablement d'un fils de Louis Hertel et de Catherine d'Aillebout, qui épousa Marie-Hippolyte Céloron de Blainville, à Laprairie, le 10 octobre 1757.

Je vois qu'un Hertel de Saint-François, autre que le Chevalier Hertel, a été fait enseigne en second en 1756, puis enseigne en pied, le ler janvier 1759, à la place du sieur de Saint-Blin l'aîné. ${ }^{90}$ Sa commission d'enseigne en pied, signée par Louis XV, se trouve aux archives de la Société historique de Chicago. Je crois qu'il s'agit, cette fois, de notre Louis-Joseph.

Le 5 mars 1764, Louis-Joseph Hertel, "lieutenant dans les troupes de la Marine", était présent au mariage de Joseph Lefebvre de Beaulac et de Julie Gamelin dans l'église de SaintFrançois-du-Lac. De quand datait sa lieutenance et par qui lui avait-elle été accordée? Je ne connais pas de document me permettant de répondre. Et, après 1764 , je perds toute trace de cet officier. Il n'assistait pas au mariage de son frère Hippolyte,

${ }^{86}$ APC, B 219: 38, 48.

87 J.-J. Lefebvre, "Louise Réaume-Fournier-Robertson et son petitfils le colonel Daniel de Hertel", Revue d'Histoire de l'Amérique française, 12 (1958-1959): 330; F.-J. Audet, "Officiers canadiens dans l'armée anglaise", BRH, 29 (1923): 159.

88 La pièce se trouve aux archives de la Société historique de Chicago. $89 \mathrm{AC}, \mathrm{D}^{2}$, vol. 3 .

90 AC, D2, vol. 3 ; RAPQ (1923-1924): 38; APC, Collection Bourlamaque, vol. $6: 339$. 
à Montréal le 24 août 1767, ni à celui de sa sœur Catherine avec Ignace de Salaberry, à Montréal encore, le 18 février 1778.

Reste Jean-Baptiste, le seul qu'on puisse identifier avec le Hertel de Saint-François qui fut tué à Chouaguen, en 1759. Le chevalier de LaCorne avait été invité par les Iroquois des CinqNations à se porter sur la rivière Chouaguen, où les Anglais commençaient à faire des mouvements. Le 6 juillet, à la pointe $\mathrm{du}$ jour, il se disposa à investir les ennemis dans leur retranchement, afin de faciliter la besogne de ses berges. La fusillade dura une heure. Il y perdit dix hommes, dont l'enseigne Hertel de Saint-François. ${ }^{91}$ Ce dernier n'avait que quatorze ans.

Comme on le voit, l'héroïsme de François Hertel bouillait encore dans le sang de ses petits-enfants. Joseph Hertel de SaintFrançois n'avait sûrement pas présumé de leur bravoure en écrivant au gouverneur La Galissonière, après la mort du premier: "Vous voyez, Monsieur, que je n'ai plus d'espérance que dans les cinq qui me restent, que j'ai l'honneur de vous recommander, à mesure qu'ils deviendront en état de servir. J'ai même très lieu d'être content du soin que vous en avez pris jusqu'à présent. Ils promettent beaucoup."

Il n'y a plus de Hertel à Saint-François. Seul le nom subsiste, donné à un des chenaux qui longent l'île Saint-Jean, où sont nés les six frères. La grande histoire elle-même n'a retenu que le nom de leur tante, cette dame de Beaubassin dont la compagnie réjouissait tant le cœur du grand vainqueur de Carillon.

Thomas ChaRLaNd

${ }^{91}$ APC, rapport pour 1886: CLXXIX; Relation de la campagne de M. le chevalier de LaCorne à Chouaguen en 1759, CMML, 11: 217-218; “Journal d'Avène des Méloizes", RAPQ (1928-1929): 50. 